\title{
Identifying of Mungbean Resistance Genotypes against Mungbean Yellow Mosaic Virus, Anthracnose and Cercospora Diseases under Natural Condition in Tripura
}

\author{
Debashre Bhattacharjee $^{1^{*}}$ and Jayashree Bhattacharjee ${ }^{2}$
}

${ }^{1}$ MULLaRP, ICAR RC for NEH Region, Tripura Centre, Lambucherra-799210

${ }^{2}$ Department of Plant Pathology, Bidhan Chandra KrishiViswavidyalaya, Mohanpur, Nadia, West Bengal-741252

*Corresponding author

\section{A B S T R A C T}

\section{Keywords}

Germplasm,

Mungbean,

Resistance,

Screening, Yellow

Mosaic Virus,

Cercospora,

Anthracnose

Article Info

Accepted:

04 August 2019

Available Online:

10 September 2019
One hundred one genotypes/lines of mungbean germplasm were screened against MYMV, cercospora and anthracnose under natural field conditions at Tripura centre lembucherra. The germplasm was categorized in to resistant and susceptible depending upon severity of disease. The present investigation aimed to identify stable both MYMV, cercospora and anthracnose resistant lines with high yielding through screening under natural condition. The experimental material consisted of 101 germplasm lines screened under field condition. Results revealed that most of the genotypes studied were categorized as resistant to moderately resistant. In spite of the variable response to MYMV, Cercospora and Anthracnose TRCM131-1, TRCM83-23, TRCM1-9-3, TRCM3-1-6, TRCM2-4-2, TRCM5-6-2, TRCM3-6-1, TRCM2-4-3, TRCM81-2-1, TRCM1-8-2, TRCM7-1-3, TRCM3-5-1, TRCM13-4-3, TRCM13-4-1, TRCM2-1-2, TRCM83-2-10 best genotypes with higher yield would be utilized as donors to develop resistant lines and these will be advanced for multilocational yield trial for selection as promising variety.

\section{Introduction}

Mungbean (Vigna radiate L. Wilczek) is one of the important pulse crops in India. Among several constraints for mungbean production, Mungbean Yellow Mosaic Virus (MYMV), anthracnose and cercospora diseases are occupies prime position and the most destructive and devastating viral disease. MYMV transmitted through whitefly (Bemisia tabaci). Spread of MYMV incidence is only possible by way of controlling the vector viz., whitefly population using insecticides which are ineffective under severe infestations. Yield loss up to $80 \%$ was reported in susceptible cultivars by Ayub et al., (1989). Mungbean is affected with different fungal, bacterial and viral diseases. (Singh, 2011) but viral diseases are serious threat to crop and among them, yellow mosaic disease caused by mungbean 
yellow mosaic virus (MYMV) appeared to be serious and widely spread in Pakistan, Bangladesh, India, Srilanka, Thailand, Philippines (Bakar 1981; Honda et al., 1983; Chenulu and Verma, 1988; Malik 1991; Malik and Bashir, 1992). Mungbean anthracnose caused by a fungal pathogen Colletotrichum lindemuthianum (sacc. and magns.).

Use of virus resistant variety is the only solution to avoid occurrence of MYMV disease.As well as Cercospora leaf spot and anthracnose disease of mungbean is a destructive and widespread fungal disease. The present investigation aimed to identify stable MYMV anthracnose and cercospora resistant lines through screening under natural condition. The experimental material consisted of 101 stable advanced breeding lines developed at ICAR Research Complex for NEH Region, Tripura Centre, following pedigree selection from different crosses. The screening was carried out under normal field condition at two locations during kharif, 2015. Infected rows with most susceptible variety were planted along with the test entries. Results revealed that most of the developed breeding lines were moderately resistant to resistant in reaction to MYMV. In spite of the variable response to MYMV, the genotypes TRCM 415-1-1, TRCM 2-4-2, TRCM 86-2-3, TRCM 1-3-1, TRCM 1-6-5-4, TRCM 7-8-12, TRCM 3-5-1, TRCM 3-3-5, TRCM 7-1lexhibited resistance in both the locations and gives highest yield. These genotypes would be utilized as donors to develop MYMV resistant lines.

\section{Materials and Methods}

The experimental material in the present study consisted of 101 mungbean germplasm lines collected from ICAR Tripura centre. The above materials were screened under natural condition to yellow mosaic virus anthracnose and cercospora disease. Each entry is sown in two lines of three meter length with the spacing of $25 \times 10 \mathrm{~cm}$ in two replications. All the recommended agronomic practices were followed. No insecticidal and fungicidal spray was given in order to allow the whitefly and fungal population to spread the diseases. Disease incidence was recorded periodically.

\begin{tabular}{|l|l|l|l|}
\hline $\begin{array}{l}\text { Disease } \\
\text { severity }\end{array}$ & Percent infection & Infection category & Reaction group \\
\hline $\mathbf{1}$ & No infection & Resistant & RR \\
\hline $\mathbf{3}$ & 11-20\%infection & Moderately resistant & MR \\
\hline $\mathbf{5}$ & 21-30\%infetion & Moderately Susceptible & MS \\
\hline $\mathbf{7}$ & $30-50 \%$ infection & susceptible & S \\
\hline $\mathbf{9}$ & Morethan 50\%infection & Highly susceptible & HS \\
\hline
\end{tabular}

Breeding lines were graded as no Infection/ highly resistant (1/R), 11-20\% plants tissue infection (3/MR), highly moderately resistant (5/MS), susceptible (7/S) and highly susceptible (9/HS) reaction, respectively.

Resistant category with higher yield will be advanced for multilocational yield trial for selection as promising variety. Other lines with resistant reaction to MYMV anthracnose and cercospora will be utilized in further improvement.

\section{Results and Discussion}

Evolution of resistant varieties is considered to be the most feasible and durable solution of controlling of MYMV diseases and as well as cercospora and anthracnose diseases under natural condition without spraying any insecticidal and fungicidal spray (Table 1). 
Table.1 Per se performance of the advanced breeding lines of mung for yield and yield attributing characters

\begin{tabular}{|c|c|c|c|c|c|c|c|c|}
\hline Grade & $\begin{array}{l}\text { YMV } \\
\text { disease } \\
\text { score }\end{array}$ & Genotypes & Grade & $\begin{array}{l}\text { Cercospora } \\
\text { disease } \\
\text { scoring }\end{array}$ & Genotypes & Grade & Anthracnose & Genotypes \\
\hline $\mathbf{R}$ & 1 & TRCM131-1 & $\mathrm{R}$ & 1 & TRCM131-1 & $\mathrm{R}$ & 1 & TRCM131-1 \\
\hline $\mathbf{R}$ & 1 & TRCM85-2-1 & $\mathrm{R}$ & 1 & TRCM1-7-60 & $\mathrm{R}$ & 1 & TRCM85-2-1 \\
\hline $\mathbf{R}$ & 1 & TRCM415-1-1 & $\mathrm{R}$ & 1 & TRCM5-1-1 & $\mathrm{R}$ & 1 & TRCM83-2-3 \\
\hline $\mathbf{R}$ & 1 & TRCM403-1-1 & $\mathrm{R}$ & 1 & TRCM407-1 & $\mathrm{R}$ & 1 & TRCM1-9-4 \\
\hline $\mathbf{R}$ & 1 & TRCM2-3-1 & $\mathrm{R}$ & 1 & TRCM104-4-3 & $\mathrm{R}$ & 1 & TRCM1-9-3 \\
\hline $\mathbf{R}$ & 1 & TRCM1-1-1 & $\mathrm{R}$ & 1 & TRCM83-2-3 & $\mathrm{R}$ & 1 & TRCM3-1-6 \\
\hline $\mathbf{R}$ & 1 & TRCM1-8-5 & $\mathrm{R}$ & 1 & TRCM1-9-3 & $\mathrm{R}$ & 1 & TRCM1-9-1 \\
\hline $\mathbf{R}$ & 1 & TRCM1-7-3 & $\mathrm{R}$ & 1 & TRCM1-2-1(4 lines) & $\mathrm{R}$ & 1 & TRCM2-7-1 \\
\hline $\mathbf{R}$ & 1 & TRCM1-7-4 & $\mathrm{R}$ & 1 & TRCM103-1 & $\mathrm{R}$ & 1 & TRCM1-6-2 \\
\hline $\mathbf{R}$ & 1 & TRCM9-3-3 & $\mathrm{R}$ & 1 & TRCM3-1-6 & $\mathrm{R}$ & 1 & TRCM88-3-3 \\
\hline $\mathbf{R}$ & 1 & TRCM1-7-60 & $\mathrm{R}$ & 1 & TRCM1-9-1 & $\mathrm{R}$ & 1 & TRCM2-4-2 \\
\hline $\mathbf{R}$ & 1 & TRCM5-1-1 & $\mathrm{R}$ & 1 & TRCM2-7-1 & $\mathrm{R}$ & 1 & $\begin{array}{l}\text { TRCM86-2-3 } \\
\text { (4line }\end{array}$ \\
\hline $\mathbf{R}$ & 1 & TRCM407-1 & $\mathrm{R}$ & 1 & TRCM88-3-3 & $\mathrm{R}$ & 1 & TRCM1-3-5 \\
\hline $\mathbf{R}$ & 1 & TRCM104-4-3 & $\mathrm{R}$ & 1 & TRCM2-4-2 & $\mathrm{R}$ & 1 & TRCM5-6-2 \\
\hline $\mathbf{R}$ & 1 & TRCM83-2-3 & $\mathrm{R}$ & 1 & TRCM86-2-3(4line & $\mathrm{R}$ & 1 & TRCM3-1-1 \\
\hline $\mathbf{R}$ & 1 & TRCM1-9-3 & $\mathrm{R}$ & 1 & TRCM1-3-1 & $\mathrm{R}$ & 1 & TRCM2-7-7 \\
\hline $\mathbf{R}$ & 1 & TRCM1-2-1 & $\mathrm{R}$ & 1 & TRCM5-6-2 & $\mathrm{R}$ & 1 & TRCM3-6-1 \\
\hline $\mathbf{R}$ & 1 & TRCM103-1 & $\mathrm{R}$ & 1 & TRCM1-6-5-4 & $\mathrm{R}$ & 1 & TRCM2-4-3 \\
\hline $\mathbf{R}$ & & TRCM3-1-6 & $\mathrm{R}$ & 1 & TRCM1-3-3 & $\mathrm{R}$ & 1 & TRCM5-4-2 \\
\hline $\mathbf{R}$ & 1 & TRCM1-9-1 & $\mathrm{R}$ & & TRCM1-1-2 & $\mathrm{R}$ & 1 & TRCM81-2-1 \\
\hline $\mathbf{R}$ & 1 & TRCM2-7-1 & $\mathrm{R}$ & 1 & TRCM74-2-3 & $\mathrm{R}$ & 1 & TRCM1-8-2 \\
\hline $\mathbf{R}$ & 1 & TRCM88-3-3 & $\mathrm{R}$ & 1 & TRCM2-2-1 & $\mathrm{R}$ & 1 & TRCM151-1 \\
\hline $\mathbf{R}$ & 1 & TRCM2-4-2 & $\mathrm{R}$ & 1 & TRCM3-1-1 & $\mathrm{R}$ & 1 & TRCM2-3-5 \\
\hline $\mathbf{R}$ & 1 & TRCM86-2-3 & $\mathrm{R}$ & 1 & TRCM7-8-12 & $\mathrm{R}$ & 1 & $\begin{array}{l}\text { TRCM7-1-3(4 } \\
\text { line) }\end{array}$ \\
\hline $\mathbf{R}$ & 1 & TRCM1-3-1 & $\mathrm{R}$ & 1 & TRCM1-6-3 & $\mathrm{R}$ & 1 & TRCM3-5-1 \\
\hline $\mathbf{R}$ & & TRCM5-6-2 & $\mathrm{R}$ & 1 & TRCM3-4-4 & $\mathrm{R}$ & 1 & TRCM13-4-3 \\
\hline $\mathbf{R}$ & 1 & TRCM1-6-5-4 & $\mathrm{R}$ & 1 & TRCM2-7-7 & $\mathrm{R}$ & 1 & TRCM1-8-4 \\
\hline $\mathbf{R}$ & 1 & TRCM1-3-3 & $\mathrm{R}$ & 1 & TRCM3-6-1 & $\mathrm{R}$ & 1 & TRCM2-7-2 \\
\hline $\mathbf{R}$ & 1 & TRCM74-2-3 & $\mathrm{R}$ & 1 & TRCM1-4-2 & $\mathrm{R}$ & 1 & TRCM9-4-7 \\
\hline $\mathbf{R}$ & 1 & TRCM7-8-12 & $\mathrm{R}$ & 1 & TRCM2-7-5 & $\mathrm{R}$ & 1 & TRCM3-1-2 \\
\hline $\mathbf{R}$ & 1 & TRCM3-6-1 & $\mathrm{R}$ & 1 & TRCM2-4-3 & $\mathrm{R}$ & 1 & TRCM2-1-3 \\
\hline $\mathbf{R}$ & 1 & TRCM2-4-3 & $\mathrm{R}$ & 1 & TRCM7-2-1(4line) & $\mathrm{R}$ & 1 & $\begin{array}{l}\text { TRCM5-1- } \\
\text { 2(4line) }\end{array}$ \\
\hline $\mathbf{R}$ & 1 & TRCM1-9-5 & $\mathrm{R}$ & 1 & TRCM5-4-2 & $\mathrm{R}$ & 1 & TRCM303-4-1 \\
\hline $\mathbf{R}$ & 1 & TRCM88-3-2 & $\mathrm{R}$ & 1 & TRCM81-2-1 & $\mathrm{R}$ & 1 & TRCM2-3-1 \\
\hline $\mathbf{R}$ & 1 & TRCM5-4-2 & $\mathrm{R}$ & 1 & TRCM1-8-2 & $\mathrm{R}$ & 1 & TRCM13-4-1 \\
\hline $\mathbf{R}$ & 1 & TRCM81-2-1 & $\mathrm{R}$ & 1 & TRCM1-7-60 & $\mathrm{R}$ & 1 & TRCM4-4-3 \\
\hline $\mathbf{R}$ & 1 & TRCM1-8-2 & $\mathrm{R}$ & 1 & TRCM151-1 & $\mathrm{R}$ & 1 & TRCM7-2-2 \\
\hline $\mathbf{R}$ & 1 & TRCM1-7-60 & $\mathrm{R}$ & 1 & TRCM1-5-1 & $\mathrm{R}$ & 1 & TRCM1-6-4 \\
\hline $\mathbf{R}$ & 1 & TRCM1-5-1 & $\mathrm{R}$ & 1 & TRCM2-3-5 & $\mathrm{R}$ & 1 & TRCM83-2-10 \\
\hline $\mathbf{R}$ & 1 & TRCM2-8-2 & $\mathrm{R}$ & 1 & TRCM3-4-2 & $\mathrm{R}$ & 1 & TRCM2-1-2 \\
\hline $\mathbf{R}$ & 1 & TRCM7-1-3 & $\mathrm{R}$ & 1 & TRCM2-8-2 & $\mathrm{R}$ & 1 & TRCM7-1-1 \\
\hline $\mathbf{R}$ & 1 & TRCM3-5-1 & $\mathrm{R}$ & 1 & TRCM7-1-3 & $\mathrm{R}$ & 1 & TRCM100-5-2 \\
\hline
\end{tabular}




\begin{tabular}{|c|c|c|c|c|c|c|c|c|}
\hline & & & & & (4 line) & & & \\
\hline $\mathbf{R}$ & 1 & TRCM2-6-3 & $\mathrm{R}$ & 1 & TRCM3-5-1 & MR & 3 & $\begin{array}{l}\text { TRCM415-1- } \\
1 \text { (6 lines) }\end{array}$ \\
\hline $\mathbf{R}$ & 1 & TRCM5-2-2 & $\mathrm{R}$ & 1 & TRCM1-1-4(4 line) & MR & 3 & TRCM403-1-1 \\
\hline $\mathbf{R}$ & 1 & TRCM1-5-3 & $\mathrm{R}$ & 1 & TRCM2-6-3 & MR & 3 & TRCM2-3-1 \\
\hline $\mathbf{R}$ & 1 & TRCM3-6-5 & $\mathrm{R}$ & 1 & TRCM5-2-2 & MR & 3 & TRCM1-1-1 \\
\hline $\mathbf{R}$ & 1 & TRCM13-4-3 & $\mathrm{R}$ & 1 & TRCM1-5-3 & MR & 3 & TRCM1-8-5 \\
\hline $\mathbf{R}$ & 1 & TRCM3-5-1 & $\mathrm{R}$ & 1 & TRCM3-6-5 & MR & 3 & TRCM1-7-3 \\
\hline $\mathbf{R}$ & 1 & TRCM2-7-2 & $\mathrm{R}$ & 1 & TRCM13-4-3 & MR & 3 & $\begin{array}{l}\text { TRCM1-2-1(4 } \\
\text { lines) }\end{array}$ \\
\hline $\mathbf{R}$ & 1 & TRCM2-7-9 & $\mathrm{R}$ & 1 & TRCM1-4-1 & MR & 3 & TRCM103-1 \\
\hline $\mathbf{R}$ & 1 & TRCM9-4-7 & $\mathrm{R}$ & 1 & TRCM2-7-4 & MR & 3 & TRCM1-2-2 \\
\hline $\mathbf{R}$ & 1 & TRCM3-6-3 & $\mathrm{R}$ & 1 & TRCM7-4-8(6 lines) & MR & 3 & TRCM1-3-1 \\
\hline $\mathbf{R}$ & 1 & TRCM3-1-2 & $\mathrm{R}$ & 1 & TRCM1-8-4 & MR & 3 & TRCM1-6-5-4 \\
\hline $\mathbf{R}$ & 1 & TRCM303-4-1 & $\mathrm{R}$ & 1 & TRCM3-5-1 & MR & 3 & TRCM1-1-2 \\
\hline $\mathbf{R}$ & 1 & TRCM2-3-1 & $\mathrm{R}$ & 1 & TRCM2-7-2 & MR & 3 & TRCM74-2-3 \\
\hline $\mathbf{R}$ & 1 & TRCM13-4-1 & $\mathrm{R}$ & 1 & TRCM2-7-9 & MR & 3 & TRCM2-2-1 \\
\hline $\mathbf{R}$ & 1 & TRCM4-4-3 & $\mathrm{R}$ & 1 & TRCM9-4-7 & MR & 3 & TRCM7-8-12 \\
\hline $\mathbf{R}$ & 1 & TRCM303-3-2 & $\mathrm{R}$ & 1 & TRCM3-6-3 & MR & 3 & TRCM1-6-3 \\
\hline $\mathbf{R}$ & 1 & TRCM8-2-10 & $\mathrm{R}$ & 1 & TRCM3-1-2 & MR & 3 & TRCM3-4-4 \\
\hline $\mathbf{R}$ & 1 & TRCM3-3-5 & $\mathrm{R}$ & 1 & TRCM2-1-3 & MR & 3 & TRCM1-4-2 \\
\hline $\mathbf{R}$ & 1 & TRCM7-2-2 & $\mathrm{R}$ & 1 & TRCM5-1-2(4line) & MR & 3 & TRCM2-7-5 \\
\hline $\mathbf{R}$ & 1 & TRCM1-6-4 & $\mathrm{R}$ & 1 & TRCM303-4-1 & MR & 3 & $\begin{array}{l}\text { TRCM7-2- } \\
\text { 1(4line) }\end{array}$ \\
\hline $\mathbf{R}$ & 1 & TRCM3-1-5 & $\mathrm{R}$ & 1 & TRCM2-3-1 & MR & 3 & TRCM7-3-1 \\
\hline $\mathbf{R}$ & 1 & TRCM7-1-2 & $\mathrm{R}$ & 1 & TRCM13-4-1 & MR & 3 & TRCM1-9-5 \\
\hline $\mathbf{R}$ & 1 & TRCM83-2-10 & $\mathrm{R}$ & 1 & TRCM4-4-3 & MR & 3 & TRCM88-3-2 \\
\hline $\mathbf{R}$ & 1 & TRCM2-1-2 & $\mathrm{R}$ & 1 & TRCM303-3-2 & MR & 3 & TRCM1-5-1 \\
\hline $\mathbf{R}$ & 1 & TRCM7-1-1 & $\mathrm{R}$ & 1 & TRCM8-2-10 & MR & 3 & TRCM2-8-2 \\
\hline $\mathbf{R}$ & 1 & TRCM8-2-5 & $\mathrm{R}$ & 1 & TRCM3-3-5 & MR & 3 & $\begin{array}{l}\text { TRCM1-1-4(4 } \\
\text { line) }\end{array}$ \\
\hline $\mathbf{R}$ & 1 & TRCM100-5-2 & $\mathrm{R}$ & & TRCM1-6-4 & MR & 3 & TRCM1-5-3 \\
\hline MR & 3 & TRCM1-4-4 & $\mathrm{R}$ & 1 & TRCM1-9-1 & MR & 3 & TRCM1-4-1 \\
\hline MR & 3 & TRCM1-8-3 & $\mathrm{R}$ & 1 & TRCM7-1-2 & MR & 3 & TRCM2-7-4 \\
\hline MR & 3 & TRCM4-7-1 & $\mathrm{R}$ & 1 & TRCM8-2-6 & MR & 3 & $\begin{array}{l}\text { TRCM7-4-8(6 } \\
\text { lines) }\end{array}$ \\
\hline MR & 3 & TRCM1-9-4 & $\mathrm{R}$ & 1 & TRCM83-2-10 & MR & 3 & TRCM3-5-1 \\
\hline MR & 3 & TRCM1-6-2 & $\mathrm{R}$ & 1 & TRCM2-1-2 & MR & 3 & TRCM2-7-9 \\
\hline MR & 3 & TRCM1-2-2 & $\mathrm{R}$ & 1 & TRCM100-5-2 & MR & 3 & TRCM3-6-3 \\
\hline MR & 3 & TRCM1-3-5 & $\mathrm{R}$ & 1 & TRCM5-2-5 & MR & 3 & TRCM8-2-10 \\
\hline MR & 3 & TRCM1-1-2 & MR & 3 & TRCM85-2-1 & MR & 3 & TRCM3-3-5 \\
\hline MR & 3 & TRCM2-2-1 & MR & 3 & $\begin{array}{l}\text { TRCM415-1-1(6 } \\
\text { lines) }\end{array}$ & MR & 3 & TRCM86-2-9 \\
\hline MR & 3 & TRCM3-1-1 & MR & 3 & TRCM403-1-1 & MR & 3 & TRCM3-1-5 \\
\hline MR & 3 & TRCM1-6-3 & MR & 3 & TRCM1-1-1 & MR & 3 & TRCM1-9-1 \\
\hline MR & 3 & TRCM3-4-4 & MR & 3 & TRCM1-8-5 & MR & 3 & TRCM7-1-2 \\
\hline MR & 3 & TRCM2-7-5 & MR & 3 & TRCM1-8-3 & MR & 3 & TRCM5-2-5 \\
\hline MR & 3 & TRCM7-2-1 & MR & 3 & TRCM4-7-1 & MS & 5 & TRCM1-7-4 \\
\hline MR & 3 & TRCM7-3-1 & MR & 3 & TRCM1-9-4 & MS & 5 & TRCM9-3-3 \\
\hline MR & 3 & TRCM151-1 & MR & 3 & TRCM1-6-2 & MS & 5 & TRCM1-4-4 \\
\hline MR & 3 & TRCM3-4-2 & MR & 3 & TRCM1-2-2 & MS & 5 & TRCM1-7-60 \\
\hline MR & 3 & TRCM1-1-4 & MR & 3 & TRCM1-3-5 & MS & 5 & TRCM407-1 \\
\hline
\end{tabular}




\begin{tabular}{|l|l|l|l|l|l|l|l|l|}
\hline MR & 3 & TRCM1-4-1 & MR & 3 & TRCM7-3-1 & MS & 5 & TRCM1-8-3 \\
\hline MR & 3 & TRCM2-7-4 & MR & 3 & TRCM86-2-9 & MS & 5 & TRCM4-7-1 \\
\hline MR & 3 & TRCM7-4-8 & MR & 3 & TRCM7-2-2 & MS & 5 & TRCM104-4-3 \\
\hline MR & 3 & TRCM1-8-4 & MR & 3 & TRCM3-1-5 & MS & 5 & TRCM1-7-60 \\
\hline MR & 3 & TRCM2-1-3 & MS & 5 & TRCM2-3-1 & MS & 5 & TRCM1-3-3 \\
\hline MR & 3 & TRCM5-1-2 & MS & 5 & TRCM1-7-3 & MS & 5 & TRCM3-4-2 \\
\hline MR & 3 & TRCM86-2-9 & MS & 5 & TRCM9-3-3 & MS & 5 & TRCM5-2-2 \\
\hline MR & 3 & TRCM1-9-1 & MS & 5 & TRCM1-4-4 & MS & 5 & TRCM3-6-5 \\
\hline MR & 3 & TRCM8-2-6 & MS & 5 & TRCM88-3-2 & MS & 5 & TRCM8-2-6 \\
\hline MR & 3 & TRCM5-2-5 & MS & 5 & TRCM7-1-1 & MS & 5 & TRCM8-2-5 \\
\hline MS & 5 & TRCM2-7-7 & MS & 5 & TRCM8-2-5 & S & 7 & TRCM2-6-3 \\
\hline MS & 5 & TRCM1-4-2 & S & 7 & TRCM1-7-4 & S & 7 & TRCM303-3-2 \\
\hline MS & 5 & TRCM2-3-5 & S & 7 & TRCM1-9-5 & HS & 9 & TRCM5-1-1 \\
\hline
\end{tabular}

Screening mungbean germplasm against these diseases under natural condition is the first step in identifying the resistant donors for evolving the mungbean varieties with YMV, anthracnose and cercospora resistance.

The study revealed that maximum number of entries was grouped under Resistant to Moderately resistant categories in both the locations. Out of 101 breeding lines tested, 72 exhibited resistant, 26 moderately resistant, and only 3moderately susceptible disease reaction in case of ymv disease. Naqvi et al., (1995) studied that out of 10 tested lines, there was no resistant line to MYMV. Shad et al (2006) found that there was no resistant line against MYMV and identification of seven susceptible and 247 as highly susceptible lines exhibited meager resistance in mungbean. Iqbal et al., (2011) screened 100 lines of mungbean germplasm and out of which only four lines shows resistance under field condition. Salam et al., (2009) found 3 lines out of 93 genotypes as resistant.

Habib et al., (2007) evaluated 108 germplasm lines but no resistant line was found. In our study while screening of cercospora disease 78 lines exhibited to cercospora15 germplasm exhibited moderately resistant, 7 lines moderately susceptible. While anthracnose disease scoring 41 lines showed, 40 moderately resistant and 15. moderately susceptible 1 susceptible and 1 highly susceptible. According to this study 29 lines resistant to ymv as well as cercospora and anthracnose diseases as considered as best promising lines, these lines are TRCM131-1, TRCM83-2-3, TRCM1-9-3， TRCM3-1-6, TRCM1-9-1, TRCM2-7-1, TRCM88-3-3, TRCM2-4-2, TRCM86-2-3， TRCM5-6-2, TRCM2-4-3, TRCM3-6-1, TRCM5-4-2, TRCM81-2-1, TRCM1-8-2, TRCM7-1-3, TRCM3-5-1, TRCM13-4-3, TRCM2-7-2, TRCM9-4-7, TRCM3-1-2, TRCM303-4-1, TRCM2-3-1, TRCM13-4-1, TRCM4-4-3, TRCM1-6-4, TRCM83-2-10, TRCM2-1-2, TRCM2-1-2 are showing resistant to YMV, cercospora, anthracnose diseases. Datta et al., (2012) also reported the resistance nature of the genotype IPM-02-03. Similarly Paul et al., (2013) reported PDM-139 (Samrat) as variety resistant to yellow mosaic and recommended for use in disease resistance breeding programmes. The genotypes grouped under resistant category with higher yield germplasms are TRCM131-1, TRCM83-2-3, TRCM1-9-3, TRCM3-1-6, TRCM2-4-2, TRCM5-6-2, TRCM3-6-1, TRCM2-4-3, TRCM81-2-1, TRCM1-8-2, TRCM7-1-3, TRCM3-5-1, TRCM13-4-3, TRCM13-4-1, TRCM2-1-2, TRCM83-2-10. These will be advanced for multi-locational yield trial for selection as promising variety. Other lines with resistant reaction to MYMV will be utilized in further improvement. For additional collaboration, these genotypes will be screened through artificial screening 
methods like forced feeding method, agroinoculation method, etc., to confirm resistance against MYMV.

In conclusion, this study identified some resistant the lines. TRCM131-1, TRCM83-23, TRCM1-9-3, TRCM3-1-6, TRCM1-9-1, TRCM2-7-1, TRCM88-3-3, TRCM2-4-2, TRCM86-2-3, TRCM5-6-2, TRCM2-4-3, TRCM3-6-1, TRCM5-4-2, TRCM81-2-1, TRCM1-8-2, TRCM7-1-3, TRCM3-5-1, TRCM13-4-3, TRCM2-7-2, TRCM9-4-7, TRCM3-1-2, TRCM303-4-1, TRCM2-3-1, TRCM13-4-1, TRCM4-4-3, TRCM1-6-4, TRCM83-2-10, TRCM2-1-2, TRCM2-1-2, which may be useful in breeding programs aimed at development of diseases free resistant cultivars. These resistant lines performed well and so can be used for development of ymv cercospora as well as anthacnose resistant moongbean cultivars without fear of losing agronomic performance. In this study, disease symptoms, disease effect on some important plant traits, and morphology of the pathogen were characterized; these traits may serve as a reference for future studies regarding moongbean and its reaction to $y m v$ cercospora and anthracnose diseases.

The genotypes grouped under resistant category would be utilized as donors to develop MYMV cercospora $\mathrm{n}$ anthracnose resistant lines. For additional corroboration, these genotypes will be screened through artificial screening methods like forced feeding method14, agroinoculation method, etc., to confirm resistance against MYMV.

\section{References}

Asthana AN. Pulse crops research in India. Indian J Agril Sci.; 68(8): 448-52, (1998).

Ayub MA, Ilyas MB, BhattiMAR. Growth responces of mungbean cultivars to mungbean yellow mosaic virus infection. Pak. J. Phytopathology, 1: 38-42, (1989).

Bakar, A.K. Pest and disease problems of mungbean in West Malaysia. Malaysian Agric. Jour. 53: 29-33, (1981).

Chenulu, V. V. and A. Verma. Virus and virus like diseases of pulse crops commonly grown in India. In Pulse Crops, ed. by B. Baldev, S. Ramajunam, and H. K. Jain, pp. 338-370. New Delhi, Oxford, (1981).

Datta S, Gangwar S, Kumar S, Gupta S, Rai1 R, Kaashyap M, Singh P, Chaturvedi SK, Singh BB, Nadarajan N. Genetic diversity in selected Indian Mungbean [Vignaradiata (L.) Wilczek] Cultivars Using RAPD Markers. American J Plant Sci, 3: 1085-91, (2012).

Habib S, Shad N, Javaid A, Iqbal U., Screening of mungbeangermplasm for resistance/tolerance against yellow mosaic disease. Mycopath. 5(2):89-94, (2007).

Honda, Y., M. Iwaki and Y. Saito. Mechanical transmission, purification and some properties of whitefly-borne mungbean yellow mosaic virus in Thailand. Plant Dis, 67:801-804, (1983).

Iqbal U, Iqbal SM, Afzal R, Jamal A, Farooq MA, Zahid A. Screening of Mungbean germplasm against Mungbean Yellow Mosaic Virus (MYMV) under field conditions. Pak J Phytopathol. 23(1): 48-51, (2011).

Malik, B. A. and M. Bashir. Major diseases of food legume crops of Islamic countries. Proceedings of COMSTECH NIAB International workshop of agro climatology pests and disease and their control. By Jamil, F. F and S. H. M. Naqvi. 25-38, (1992).

Malik, I. A. Breeding for resistance to MYMV and its vector in Pakistan. Mungbean yellow mosaic disease: 
Proceedings of an International Workshop. (Eds.): S.K. Green, (1991).

Naqvi, S.M., M.A. Rustamani, T. Hussain and M.A. Talpur. Relative resistance of mungbean varieties to whitefly and yellow mosaic. Proc. Pak. Zool. Conf., 15: 247-251, (1995).

Paul PC, Biswas MK, Mandal D, Pal P. Studies on host resistance of Mungbean against Mungbean Yellow Mosaic Virusin the agro-ecological condition of lateritic zone of West bengal. The Bioscan. 8(2): 583-87, (2013).

Salam SA, Patil MS, Salimath PM. Evaluation of Mungbean cultures against MYMV in Karnataka under natural conditions. Legume Res. 32(4): 286-89, (2009).

Shad N, Mughal SM, Farooq K, Bashir M. Evaluation of Mungbean germplasm for resistance against Mungbean Yellow Mosaic Begomovirus. Pak Bot. 38(2): 449-57, (2006).

Singh BB. Project coordinators report. Indian Council of Agricultural Research, Indian Institute of Pulses Research, All India Coordinated Research Project on MULLaRP, Annual Group Meet. 1113, (2011).

\section{How to cite this article:}

Debashre Bhattacharjee and Jayashree Bhattacharjee. 2019. Identifying of Mungbean Resistance Genotypes against Mungbean Yellow Mosaic Virus, Anthracnose and Cercospora Diseases under Natural Condition in Tripura. Int.J.Curr.Microbiol.App.Sci. 8(09): 74-80. doi: https://doi.org/10.20546/ijcmas.2019.809.011 